\title{
Differences in Thromboxane Production between Neonatal and Adult Platelets in Response to Arachidonic Acid and Epinephrine
}

\author{
MARIE J. STUART, JON DUSSE, DAVID A. CLARK AND RONALD W. WALENGA \\ Divisions of Pediatric Hematology-Oncology and Neonatology, Department of Pediatrics, State University \\ of New York, Upstate Medical Center, Syracuse, New York 13210
}

\begin{abstract}
Summary
In this study, we have investigated the possible role of the proaggregatory arachidonic acid (AA) metabolite thromboxane, in the impaired function of neonatal platelets. In platelet-rich plasma thromboxane production (measured by radioimmunoassay of thromboxane $B_{2}$ ) was not different between neonates and adults when stimulated by thrombin (at 0.1 or $1.0 \mathrm{U} / \mathrm{ml}$ ) or collagen $(70 \mu \mathrm{g} / \mathrm{ml})$ although neonatal platelets produced decreased thromboxane $\left(\mathrm{TBX}_{2}\right)$ postepinephrine stimulation. In response to $1 \mathrm{U} / \mathrm{ml}$ thrombin, adult and neonatal platelet-rich plasmas produced mean values of $3.41 \pm 0.35(\mathrm{SEM})$ and 3.11 \pm 0.49 pmol of $\mathrm{TXB}_{2} / 10^{6}$ platelets, respectively. Production of $\mathrm{TXB}_{2}$ in response to $0.1 \mathrm{U} / \mathrm{ml}$ thrombin was not dissimilar between neonates $(1.01 \pm 0.46 \mathrm{pmol})$ and adults $(1.04 \pm 0.38$ pmol). When collagen was used as the aggregating agent, TXB $_{2}$ production was also not significantly different with values of 2.44 \pm 0.48 and $1.90 \pm 0.46 \mathrm{pmol} / 10^{6}$ platelets produced by adult and neonatal platelet-rich plasma, respectively. In response to 200 $\mu \mathrm{M}$ epinephrine, adult platelets produced $1.03 \pm 0.39 \mathrm{pmol}$ $\mathrm{TXB}_{2} / \mathbf{1 0}^{6}$ platelets while neonatal platelet $T X B_{2}$ production was significantly decreased $(0.15 \pm 0.04 ; P<0.05)$. Thromboxane production in response to $\mathrm{AA}$, however, was markedly elevated in neonatal platelet-rich plasma. When 200 and $400 \mu \mathrm{M}$ concentrations of $\mathrm{AA}$ were used as the aggregating stimuli, neonatal platelet rich plasma produced $3.17 \pm 0.77$ and $8.0 \pm 1.47 \mathrm{pmol}$ $\mathrm{TXB}_{2} / 10^{6}$ platelets, respectively. These values were significantly elevated $P<0.02$ and $<0.005$ ) when compared to mean values of $0.41 \pm 0.10$ and $3.32 \pm 0.15$ pmol in adult platelet-rich plasma. This elevated thromboxane production was not, however, inherent in neonatal platelets since when washed platelets were studied, results were reversed. Adult platelets produced more thromboxane at all doses of $\mathrm{AA}$ evaluated. These results suggest that the elevated response to exogenous $\mathrm{AA}$ observed in neonatal platelet-rich plasma results from as yet undetermined plasma factors. The reported deficiencies in platelet function in the newborn clearly do not result from deficient thromboxane production poststimulation with the physiologic aggregating agents collagen and thrombin. Moreover, our study introduces a new and possibly important difference between adult and neonatal plasma, namely, the differential response to exogenous arachidonic acid.
\end{abstract}

\section{Abbreviations}

AA, arachidonic acid

$\mathbf{T X B}_{2}$, thromboxane $\mathbf{B}_{2}$

PRP, platelet-rich plasma

Received December 2, 1983

This work was supported by United States Public Health Service Grant HD 14405 and by Clinical Research Grant 6-282 from The National Foundation, The March of Dimes.

Address correspondence to: Marie J. Stuart, Professor of Pediatrics, SUNY, Upstate Medical Center, 750 East Adams Street, Syracuse, NY 13210.
An impairment in platelet function is well recognized in the neonate, and includes abnormalities in the release of storage pool adenine nucleotides, and aggregation responses to a variety of stimuli $(2,3,10)$. A previous evaluation of exogenous $\left[{ }^{14} \mathrm{C}\right] \mathrm{AA}$ metabolism in the platelet of the neonate did not suggest that neonatal platelet dysfunction is related to any specific abnormality in this pathway (13). We observed an increased release of endogenous AA from neonatal platelet membrane phospholipids, and decreased activity of neonatal platelet cyclooxygenase. These two effects counteracted each other resulting in similar conversion of prelabeled arachidonic acid to $\mathrm{TXB}_{2}$ in adult and neonatal platelets. The present study was undertaken to evaluate endogenous radioimmunoassayable $\mathrm{TXB}_{2}$ production by neonatal platelets in response to a variety to physiologic stimuli. Our results provide evidence that although there is no difference in $\mathrm{TXB}_{2}$ production between adult and neonatal platelet-rich plasmas when thrombin and collagen are used as aggregating agents, neonatal $\mathrm{TXB}_{2}$ production is decreased in response to epinephrine, and markedly increased in the presence of low doses of exogenous arachidonic acid. No such enhanced response by neonatal platelets to arachidonic acid was seen in a washed platelet system. Abnormalities in thromboxane production by neonatal platelets in response to epinephrine persisted in the washed platelet system.

\section{MATERIALS AND METHODS}

Prepartion of platelet-rich plasma or washed platelets. Umbilical cord blood samples were obtained at the time of delivery from 16 neonates (nine males and seven females). All infants weighed more than $2500 \mathrm{~g}$ and were born to healthy mothers after normal full-term pregnancies. No mother had ingested aspirin or other drugs known to affect platelet thromboxane production within 2 weeks prior to delivery. Immediately after delivery, clamps were placed on the umbilical cord, and an 18gauge needle was inserted into the umbilical vein near its placental insertion. Blood was also obtained from 13 normal healthy adults (six males and seven females) who had taken no medications for 2 weeks prior to evaluation. The study was approved by the Institutional Human Experimentation Committee.

Following informed consent, blood was drawn into a plastic syringe and immediately anticoagulated with $10 \%$ by volume of $0.1 \mathrm{M}$ buffered citrate anticoagulant. After correction for hematocrit, PRP was prepared by centrifuging the citrated blood at $200 \times g$ for $15 \mathrm{~min}$. Platelet-poor plasma was obtained by centrifugation of the remaining blood for $15 \mathrm{~min}$ at $1800 \times \mathrm{g}$. For the aggregation studies, PRP was adjusted with autologous platelet-poor plasma to a platelet count of 2.5 to $3 \times 10^{8} / \mathrm{ml}$. Platelet counts were performed using a Technicon platelet analyzer. For the experiments using washed platelets, following the washing procedure previously described (14), platelets were resuspended in Hanks' balanced salt solution containing $0.5 \mathrm{mM}$ 
calcium chloride at a concentration of 2.5 to $3 \times 10^{8} / \mathrm{ml}$. Platelet aggregation was evaluated at $37^{\circ} \mathrm{C}$ using aliquots of $0.25 \mathrm{ml} /$ aggregation experiment. The aggregating agents used in PRP included thrombin ( 1 and 0.1 units/ml; Parke-Davis, Kalamazoo, MI), acid-soluble bovine collagen ( $70 \mu \mathrm{g} / \mathrm{ml}$; Sigma), epinephrine (200 $\mu \mathrm{M}$ final concentration), and varying concentrations of arachidonic acid $(200 \mu \mathrm{M}, 400 \mu \mathrm{M}$, and $2 \mathrm{mM}$; Nucheck Prep, Elysian, MN). Aggregating agents evaluated in the washed platelet system included epinephrine $(200 \mu \mathrm{M})$ and 10,30 , and $50 \mu \mathrm{M}$ arachidonic acid. Higher concentration of AA were used in platelet-rich plasma since protein-bound arachidonic acid does not promote platelet aggregation and thromboxane formation. Following stimulation of platelet-rich plasma, or the washed platelet suspension for $5 \mathrm{~min}$ to allow for maximum $\mathrm{TXB}_{2}$ formation, the samples were diluted $1: 3$ with $25 \mathrm{mM}$ Tris-Hanks, $\mathrm{pH} 6$, to which indomethacin at a final concentration of $30 \mu \mathrm{M}$ was added. The mixture was centrifuged in an Eppendorf Microfuge at $17,000 \times g$ for $2 \mathrm{~min}$, and the supernatants were stored at $-20 \mathrm{C}^{\circ}$ until analyzed for the determination of radioimmunoassayable $\mathrm{TXB}_{2}$.

Radioimmunoassay of Thromboxane $B_{2}$. The radioimmunoassay of $\mathrm{TXB}_{2}$ was performed directly on thawed aliquots essentially as described by Granström and Kindahl (8). The antiserum to $\mathrm{TXB}_{2}$ was purchased from Seragen (Boston, MA) and used according to the manufacturers' directions except that IgG Sorb (The Enzyme Center, Cambridge, MA) was used in the final antigen-antibody complex precipitation (9). $\left[{ }^{3} \mathrm{H}\right] \mathrm{TXB}_{2}$ was purchased from Amersham, and standard $\mathrm{TXB}_{2}$ was from Upjohn, Kalamazoo, MI. This direct radioimmunoassay routinely provided linear results for $\mathrm{TXB}_{2}$ versus sample volume, and recovery of added standard in stimulated PRP or washed platelet suspensions was routinely over $90 \%$.

Albumin levels were performed on both adult and cord sera and statistical evaluations were performed by the unpaired $t$ test.

\section{RESULTS}

As depicted in Figure 1, no differences in $\mathrm{TXB}_{2}$ production by platelet-rich plasma from adults versus full term neonates was observed in response to either high or low doses of thrombin or collagen although the response to epinephrine was markedly decreased in the neonate. In response to 1 unit/ml thrombin, adult $(n=13)$ and neonatal $(n=16)$ platelet-rich plasmas produced mean values of $3.41 \pm 0.35$ (SEM) and $3.11 \pm 0.49$ pmol of $\mathrm{TXB}_{2} / 10^{6}$ platelets, respectively. Production of $\mathrm{TXB}_{2}$ in response $0.1 \mathrm{unit} / \mathrm{ml}$ thrombin was not dissimilar between neonates $(1.01 \pm 0.46 \mathrm{pmol} ; n=13)$ and adults $(1.04 \pm 0.38 \mathrm{pmol}$; $n=13$ ). When collagen was used as the aggregating agent, $\mathrm{TXB}_{2}$ production was also not significantly different with values of $2.44 \pm 0.48$ and $1.90 \pm 0.46 \mathrm{pmol} / 10^{6}$ platelets produced by adult and neonatal platelet-rich plasma, respectively. In response to $200 \mu \mathrm{M}$ epinephrine, adult platelets produced $1.03 \pm 0.39$ pmol $\mathrm{TXB}_{2} / 10^{6}$ platelets while neonatal platelet $\mathrm{TXB}_{2}$ production was significantly decreased $(0.15 \pm 0.04 ; P<0.05)$. Values of $0.04 \pm 0.007 \mathrm{pmol} / 10^{6}$ platelets were obtained in nonstimulated platelet-rich plasmas from both adults and neonates (Fig. 1).

When AA was used as the aggregating stimulus in platelet-rich plasma, neonatal $\mathrm{TXB}_{2}$ production in response to low levels of exogenous AA was significantly elevated (Fig. 2). When 200 and $400 \mu \mathrm{nM}$ AA concentrations were used as the aggregating stimuli, neonatal platelet-rich plasma produced $3.17 \pm 0.77$ and $8.0 \pm$ $1.47 \mathrm{pmol} \mathrm{TXB}_{2} / 10^{6}$ platelets, respectively. These values were significantly elevated $(P<0.02$ and $<0.005)$ when compared to mean values of $0.41 \pm 0.10$ and $3.32 \pm 0.15 \mathrm{pmol}$ in adult platelet-rich plasma. At the highest AA concentration used (2 $\mathrm{mM}), \mathrm{TXB}_{2}$ production was not dissimilar in neonatal and adult platelet-rich plasma $(16.79 \pm 0.71$ versus $14.11 \pm 1.76$ pmol, respectively). In an attempt to differentiate whether the enhanced response to exogenous arachidonic acid by neonatal platelet-rich plasma was due to differences in the neonatal platelets themselves or to plasma factors, $\mathrm{TXB}_{2}$ production in response to arachidonic acid was studied in washed platelets (Fig. 3). Enhanced $\mathrm{TXB}_{2}$ production was seen in platelets from adults rather than neonates. In response to. $50 \mu \mathrm{M} \mathrm{AA}$, washed platelets from adults $(n=7)$ produced $7.09 \pm 0.26 \mathrm{pmol} \mathrm{TXB}_{2} / 10^{6}$ platelets. This value was significantly greater $(P<0.05)$ than the mean of $3.36 \pm 0.76$ pmol obtained in platelets from the neonate $(n=7)$. Although not statistically significant, adult platelets also demonstrated elevated $\mathrm{TXB}_{2}$ production at $30 \mu \mathrm{M}$ AA $(2.58 \pm 0.45 \mathrm{pmol}$ in adults versus $1.84 \pm 0.53 \mathrm{pmol}$ in neonates), and at the lowest concentrations of arachidonic acid used $(10 \mu \mathrm{M})$ differences were significant with adult platelets producing $1.22 \pm 0.30 \mathrm{pmol}$ when compared to a mean value of $0.29 \pm 0.17 \mathrm{pmol}$ by platelets from the neonate $(P<0.025)$. Washing, however, did not correct the abnormalities in $\mathrm{TXB}_{2}$ production by neonatal platelets in response to epinephrine. Following the washing procedure, adult and neonatal platelets produced $0.03 \pm 0.01$ and $0.02 \pm 0.01$ pmol $\mathrm{TXB}_{2} / 10^{6}$ platelets, respectively.

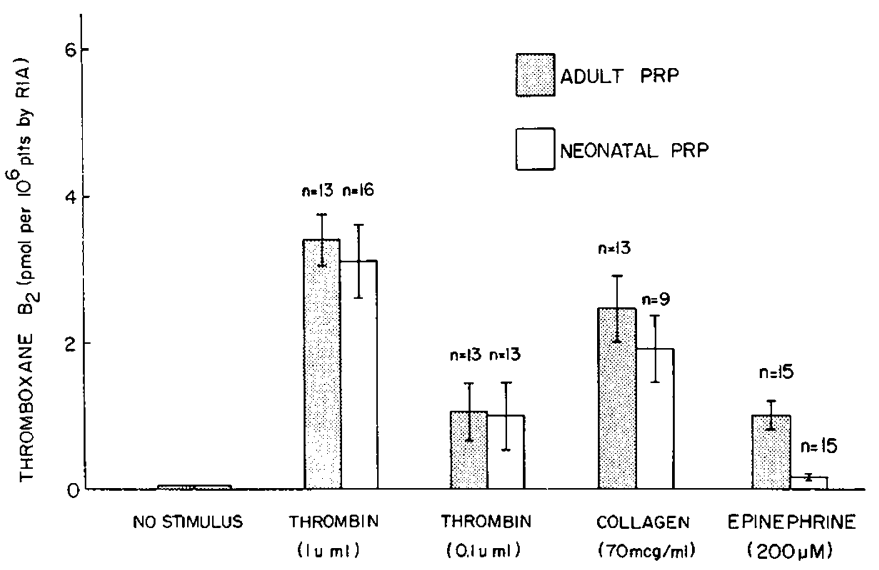

Fig. 1. Endogenous production of radioimmunoassayable $(R I A)$ thromboxane $\mathrm{B}_{2}$ by adult and neonatal unstimulated control plateletrich plasma, and following stimulation with thrombin ( 1 and 0.1 units/ $\mathrm{ml})$, bovine collagen $(70 \mu \mathrm{g} / \mathrm{ml})$, and epinephrine $(200 \mu \mathrm{M})$. Results are expressed as pmol $/ 10^{6}$ platelets, mean $\pm \mathrm{SEM}$.

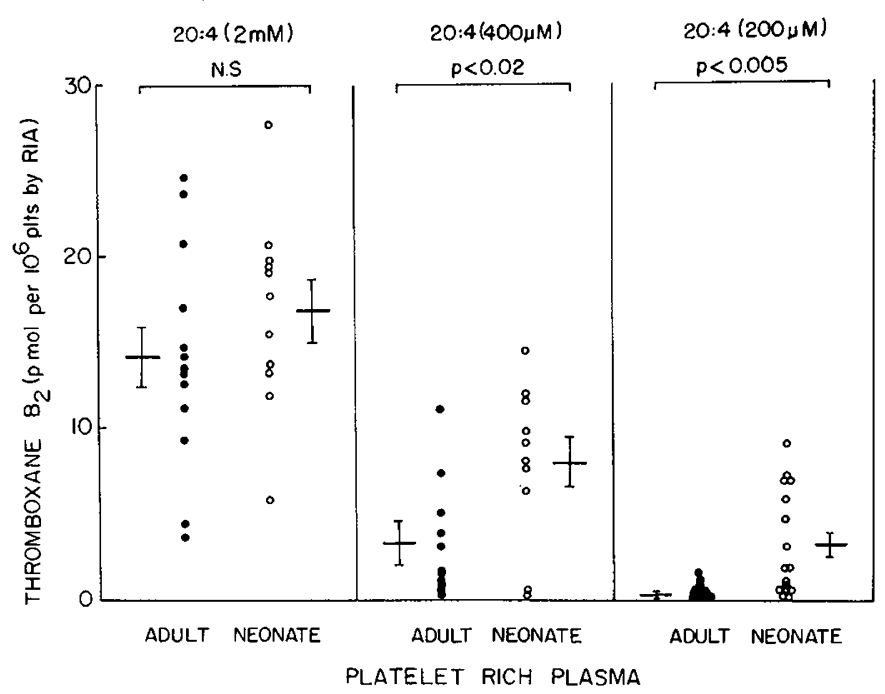

Fig. 2. Endogenous production of radioimmunoassayable $(R I A)$ thromboxane $\mathrm{B}_{2}$ by adult and neonatal platelet-rich plasma in response to various concentrations of exogenous arachidonic acid $(200 \mu \mathrm{M}, 400$ $\mu \mathrm{M}$, and $2 \mathrm{mM}$ ). Results are expressed as pmol $/ 10^{6}$ platelets, mean \pm SEM. 


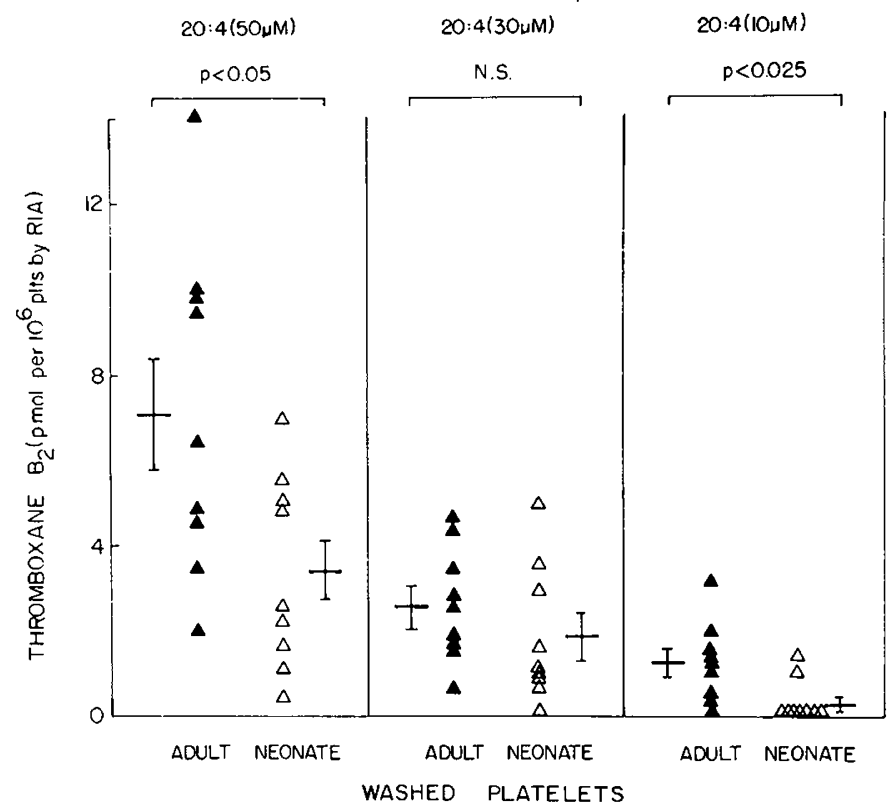

Fig. 3. Endogenous production of radioimmunoassayable ( $R I A)$ thromboxane $B_{2}$ by adult and neonatal washed platelets in response to varying concentrations of exogenous arachidonic acid (10, 30, and 50 $\mu \mathrm{M})$. Results are expressed as pmol $/ 10^{6}$ platelets, mean $\pm \mathrm{SEM}$.

No differences were observed when the adult and cord serum albumin levels were evaluated, with values of $4.20 \pm 0.07$ and $4.09 \pm 0.11 \mathrm{~g} / \mathrm{dl}$ being obtained, respectively.

\section{DISCUSSION}

Thromboxane $A_{2}$ is a potent platelet proaggregatory metabolite with multisystem effects that include vaso- and bronchoconstrictor activities. Following the release of precursor AA from platelet membrane phospholipids, this 20 -carbon polyunsaturated fatty acid is converted via the cyclic endoperoxides prostaglandins $G_{2}$ and $\mathrm{H}_{2}$ to thromboxane $\mathrm{A}_{2}$ by the platelet enzymes cyclooxygenase and thromboxane synthetase. Thromboxane $A_{2}$ is rapidly hydrolyzed to its stable end product, thromboxane $B_{2}$, which can be used as a measure of $\mathrm{TXA}_{2}$ produced during platelet activation by a variety of stimuli. Two previous studies have evaluated the formation of the various arachidonic acid metabolites in neonatal platelets, and have demonstrated production of both lipoxygenase and cyclooxygenase products by these cells $(4,13)$. In our present study, we have evaluated the abilities of both neonatal and adult platelets to produce $\mathrm{TXB}_{2}$ in response to a variety of physiologic stimuli including collagen, epinephrine, varying doses of thrombin, and arachidonic acid. $\mathrm{TXB}_{2}$ formation in platelet-rich plasma was similar when collagen and thrombin were used as the aggregating agents. In response to low levels of exogenous arachidonic aid (200 and $400 \mu \mathrm{M})$, however, neonatal $\mathrm{TXB}_{2}$ production by platelet-rich plasma was markedly enhanced. Our studies in washed platelets suggest that the cause of observed enhancement in neonatal platelet $\mathrm{TXB}_{2}$ production in response to exogenous arachidonic acid results from differences between neonatal and adult plasma. The previous study by Corby and O'Barr (4) of thromboxane formation postarachidonic acid stimulation did not find differences in metabolite production between neonatal and adult platelets. However, their study was performed in a washed platelet system.

The observed enhanced thromboxane production by plateletrich plasma from cord blood in response to low levels of exogenously provided arachidonate could be due to a variety of factors. The differences may be due to the elevated percentage of free fatty acid which arachidonate comprises in cord blood. Mean levels of endogenous free arachidonic acid in cord blood ap- proach approximately $20 \mu \mathrm{M}$ (11). Thus, elevated thromboxane production by platelet-rich plasma from cord blood in response to low levels of exogenous arachidonic acid may simply reflect elevated total (endogenous plus exogenous) free arachidonate levels in the neonate when compared with the adult. Since protein-bound arachidonate does not promote platelet responses (11), another possibility is that neonatal plasma has a decreased capacity for binding AA when compared to adult plasma. Although cord blood contains less total free fatty acids than that observed in the adult, the levels of free versus protein-bound fatty acids have not been reported.

Previous investigators have shown that hypoalbuminemia enhances platelet malondialdehyde formation (14). Hypoalbuminemia had to be relatively severe, however, with levels $<2.76 \mathrm{~g} / \mathrm{dl}$ before this effect was observed. Since we found no significant differences between the full term cord and adult serum albumin levels, differences in levels of this protein could not account for the elevated $\mathrm{TXB}_{2}$ production we have observed in neonatal platelet-rich plasma. Finally, there are other plasma components that could elevate the platelet response to exogenous arachidonate. For example, nonaggregatory levels of catecholamines reduce the concentration of arachidonic acid required to elicit aggregation in platelet-rich plasma, and enhances thromboxane production from low doses of arachidonic acid $(6,12)$. Although the specific etiology of the differential response between adult and neonatal plasma to exogenous arachidonic acid will require further elucidation, the observation is an interesting and potentially important one since arachidonic acid is the precursor of a variety of clinically important products of both the cyclooxygenase and lipoxygenase pathways including numerous important compounds, i.e., the prostaglandins, thromboxanes, and leucotrienes. Our finding that the response of platelet cyclooxygenase to low levels of arachidonic acid is increased in the plasma of the neonate could thus be associated with increased arachidonic acid availability for other vasoactive metabolite-producing cellular systems in the newborn.

Differences in platelet thromboxane production between adult and neonatal platelets were also seen when epinephrine was used as an aggregating agent, with persistence of the abnormality in the washed platelet system. Previous work by Corby and associates had demonstrated that impairment in the platelet aggregatory response to epinephrine is due to a deficiency of $\alpha$-adrenergic receptor sites in the membrane of the neonatal platelet. Thus, the marked impairment in thromboxane production by neonatal platelets in response to this catecholamine is a not unexpected finding. Since the levels of catecholamines in cord blood increase at the time of delivery (7), this markedly impaired response in production of proaggregatory platelet thromboxane in response to epinephrine may also provide a compensatory mechanism in the newborn in whom a prothrombotic tendency already exists.

Our present findings on endogenous radioimmunoassayable thromboxane $\mathbf{B}_{2}$ production are consistent with our previous study on exogenous $\left[{ }^{14} \mathrm{C}\right]$ arachidonic acid metabolism in the neonatal platelet. We had previously observed an increased release of arachidonic acid from the neonatal platelet membrane in response to thrombin, coupled with a slightly decreased activity of neonatal platelet cyclooxygenase (13). These two effects counteracted each other resulting in similar conversion of prelabeled arachidonic acid to thromboxane $\mathrm{B}_{2}$ in adult and neonatal platelets. The present observations that endogenous thromboxane production in response to thrombin and collagen is similar in adult and neonatal platelet-rich plasma, and that thromboxane production is decreased in neonatal platelets in response to exogenous arachidonate in a washed platelet system, are compatible with the results of our previous study summarized above. The additional impairment in thromboxane production by the neonatal platelet in response to epinephrine stimulation appears to be related to a membrane-associated phenomenon, i.e., the deficiency of $\alpha$-adrenergic receptor sites as previously described (5) which causes an abnormal aggregatory response in 
the neonate and thus a concomitant impairment in thromboxane production.

It is thus clear from the studies to date of arachidonate metabolism in the neonatal platelets that, although subtle differences in arachidonic acid mobilization and total cyclooxygenase activity occur, these differences do not contribute to the marked differences in aggregation and release that have been noted between platelets from the adult versus neonate, i.e., the neonatal platelet dysfunction is not "aspirin-like" in nature. Moreover, our present study introduces a new and possible important difference between adult and neonatal plasma, namely the differential response to exogenous arachidonic acid. Since this unsaturated fatty acid is the precursor of a variety of cyclooxygenase and lipoxygenase metabolites including leucotrienes produced by various cellular systems in both the adult and neonate, increased availability of this precursor compound in neonatal plasma may be of clinical importance in a variety of neonatal pathophysiologic states.

\section{REFERENCES}

1. Bills TK, Smith JB, Silver MJ 1976 Metabolism of ${ }^{14} \mathrm{C}$ arachidonic acid by human platelets. Biochim Biophys Acta 424:303
2. Corby DG, Schulman I 1972 The effects of antenatal drug administration on aggregation of platelets of newborn infants. J Pediatr 79:307

3. Corby DG, Zuck TF 1976 Newborn platelet dysfunction: a storage pool and release defect. Thromb Haemostas 36:200

4. Corby DG, O'Barr TP 1981 Neonatal platelet function: a membrane-related phenomenon? Haemostasis 10:177

5. Corby DG, O'Barr TP 1981 Decreased alpha-adrenergic receptors in newborn platelets: cause of abnormal response to epinephrine. Dev Pharm Ther 2:215

6. DiMinno G, Silver MJ, Murphy S 1982 Stored human platelets retain aggregation potential in response to pairs of aggregating agents. Blood 59:563

7. Eliot RJ, Lam R, Artal R, Leake R, Hobel C, Fisher DA 1978 Norepinephrine and epinephrine levels at birth and during the first 48 hours of life in the human Pediatr Res 12:412

8. Granström EG, Kindahl HK 1978 Radioimmunoassay of prostaglandins and thromboxane. Adv Prostaglandin Thromboxane Res 5:119

9. Goding JW 1978 Use of staphylococcal protein A as an immunological reagent. $J$ Immunol Methods 20:241

10. Mull MM, Hathaway WE 1970 Altered platelet function in newborns. Pediatr Res 4:229

11. Ogburn PL, Brenner WE 1981 The Physiologic Actions and Effects of Prostaglandins: Current Concepts. The Upjohn Co, Kalamazoo MI, p 18.

12. Rao GHR, Johnson GJ, White JG 1980 Influence of epinephrine on the aggregation response of aspirin-treated platelets. Prostaglandins Med 5:45

13. Stuart MJ, Allen JB 1982 Arachidonic acid metabolism in the neonatal platelet. J Pediatr 69:714

14. Yoshida N. Aoki N 1978 Release of arachidonic acid from human platelets. A key role for the potentiation of platelet aggregability in normal subjects and in those with nephrotic syndrome. Blood 52:969

\title{
The Influence of Early Malnutrition on Subsequent Behavioral Development. IV. Soft Neurologic Signs
}

\author{
JANINA R. GALLER, FRANK RAMSEY, GIORGIO SOLIMANO, L. THOMAS KUCHARSKI, AND \\ ROBERT HARRISON \\ Department of Child Psychiatry, Boston University School of Medicine, Boston, Massachusetts 02118 [J.R.G., \\ L.T.K.], the National Nutrition Centre, Barbados, West Indies [F.R.] the Institute of Human Nutrition and \\ Center for Population and Family Health, Columbia University, New York 10023 [G.S.], and the Department of \\ Psychology, Boston University, Boston, Massachusetts 02118 [R.H.]
}

\begin{abstract}
Summary
Soft neurologic signs were evaluated in 101 Barbadian school children, ages 4-11 years, who were malnourished in the first year of life, and 101 comparison children matched for age, sex, and handedness, but who had no history of malnutrition. Previously malnourished children performed significantly slower than comparison children on several timed motor tasks when using the nondominant hand only. Boys were found to perform significantly slower than girls, and younger (4-7 years of age) children performed slower than older (8-11 years of age) children. A model is presented that displays interrelationships among previous malnutrition, soft neurologic signs, classroom behavior, intelligence, and physical growth. In summary, slow motor per-

Received June 25, 1983; accepted December 14, 1983.

Address reprint requests to Janina R. Galler, Department of Child Psychiatry, Boston University School of Medicine, $85 \mathrm{E}$. Newton St., Boston, MA 02118.

This research was supported by grants from the Ford Foundation and the Thrasher Foundation, and was conducted in cooperation with the Ministry of Health, Barbados.
\end{abstract}

formance was associated with lower verbal and performance IQ and the presence of attention deficit disorder, as assessed by the child's teacher. The time to perform the motor tests was unrelated to measures of physical growth.

\section{Abbreviations}

WISC, Wechsler Intelligence Scale for Children MANOVA, multivariate analysis of variance MRA, multiple regression analysis

This report is one of a series describing the long-term effects of early malnutrition on the behavioral development of Barbadian school children. The earlier reports in this series demonstrated a modest reduction in IQ among the previously malnourished group (4) and the increased frequency of impaired classroom behaviors, including attention deficit disorder, impaired social skills, emotional instability, and impaired physical appearance (5). These factors resulted in poor school achieve- 